\title{
Quadrivalent Human Vaccine-A Call to Action and for Additional Research
}

$\mathrm{T}_{\mathrm{r}}$ The quadrivalent human papillomavirus (HPV) vaccine recently licensed by the U.S. Food and Drug Adminstration has been touted in the lay press as a cancer vaccine. It is both more and less than that. Infection with HPV is the most common sexually transmitted infection in the United States. It is usually acquired soon after sexual debut, and the eventual outcome depends on the type of HPV encountered (more than 100 distinct variants exist), as well as by factors specific to the host. Most HPV infections are unsuspected and asymptomatic, and resolve within 1 to $2 \mathrm{y}$. Unfortunately, many infections do not resolve, and persistent infection with certain HPV types is responsible for nearly all cervical cancers, the second most common fatal malignancy in American women. Other HPV types cause anogenital warts, low-grade cervical cytologic changes, and juvenile recurrent respiratory papillomatosis (JRPP). HPV is also involved in the vast majority of anal cancers, and there is substantial evidence that sexually acquired HPV infection may play a role in the development of oropharyngeal squamous cell cancers and other head and neck malignancies.

The current HPV vaccine contains a mixture of noninfectious virus-like particles formed by the L1 proteins from HPV types 6, 11, 16 and 18 that have been expressed in Saccharomyces cerevisiae. Types 16 and 18 are responsible for 65 to $78 \%$ of cases of cervical cancer worldwide, while types 6 and 11 are responsible for $90 \%$ of cases of genital warts and most cases of JRPP. The true efficacy of the vaccine in preventing cervical cancer will not be known for decades; approval of the vaccine was largely based on a reduction in the rate of high grade cervical intraepithelial neoplasia (CIN 2/3) and adenocarcinoma in situ. The quadrivalent vaccine was also highly effective against anogenital disease, including genital warts. This salubrious effect has received less attention, presumably because of theoretical concerns that knowledge of the activity of the vaccine, and acknowledgement of sexual activity among adolescents, could lead to disinhibition of their behavior. Although this has been characterized as an emotional and groundless sociopolitical distraction, it is true that there is much to learn about the utility and effectiveness of this vaccine, and much work to be done to lessen the burden of HPV-related disease.

The present HPV vaccine is recommended for all girls 11-12 y of age and catch up immunization for females 13-26 y of age. The goal of vaccination is to prevent infections with the vaccine serotypes and is thought to offer no benefit to persons with prior infection with these serotypes. Since 22 to $35 \%$ of cervical cancers are due to serotypes that are not covered by the current vaccine, it is important to note that vaccination is not a substitute for routine cervical cancer screening. Vaccinated females should have cervical screening as recommended. It is also important to point out that the present immunization strategy will not result in herd immunity. To have an effect on the circulation of the vaccine serotypes, universal immunization of males of similar ages will also have to occur. As a first step, studies have already been completed that demonstrate equivalent antibody titers following immunization of young girls and boys. Among other questions, the duration of protection afforded by the three injection series is currently unknown, as is the effectiveness of receiving only one or two doses. There is also concern about the possibility that less common HPV types will replace those taken out of play by the vaccine, so that there will be no net reduction of HPV related disease. Expanding the coverage of HPV vaccines to include other serotypes with malignant potential is currently under investigation, and would help address this concern. In summary, the current HPV vaccine is an excellent place to begin the foray of pediatricians into the battle against HPV related disease, but we certainly have much to learn and far to go. - Paul Krogstad and James D. Cherry.

\section{REFERENCES}

1. Markowitz LE, Dunne EF, Saraiya M, Lawson HW, Chesson H, Unger ER. Centers for Disease Control and Prevention (CDC); Advisory Committee on Immunization Practices (ACIP) 2007 Quadrivalent Human Papillomavirus Vaccine; Recommendations of the Advisory Committee on Immunization Practices (ACIP). MMWR Recomm Rep 56:1-24

2. Charo RA 2007 Politics, parents, and prophylaxis-mandating HPV vaccination in the United States. N Engl J Med 356:1905-1908 